\title{
On the intersection of tame subgroups in groups acting on
}

\author{
trees \\ Konstantinos Lentzos and Mihalis Sykiotis
}

July 30, 2021

\begin{abstract}
Let $G$ be a group acting on a tree $T$ with finite edge stabilizers of bounded order. We provide, in some very interesting cases, upper bounds for the complexity of the intersection $H \cap K$ of two tame subgroups $H$ and $K$ of $G$ in terms of the complexities of $H$ and $K$. In particular, we obtain bounds for the Kurosh rank $K r(H \cap K)$ of the intersection in terms of Kurosh ranks $K r(H)$ and $K r(K)$, in the case where $H$ and $K$ act freely on the edges of $T$.
\end{abstract}

\section{Introduction}

In 1954, Howson [9] showed that the intersection of two finitely generated subgroups $H$ and $K$ of a free group $F$ is also finitely generated and provided an upper bound for the rank $r(H \cap K)$ of $H \cap K$ in terms of $r(H)$ and $r(K)$. The Hanna Neumann conjecture, proved independently by Friedman [8] and Mineyev [12] in 2011, says that $\bar{r}(H \cap K) \leq \bar{r}(H) \bar{r}(K)$, where $\bar{r}(A)=\max \{0, r(A)-1\}$ is the reduced rank of a free group $A$.

For free products the situation is analogous. Let $\Gamma$ be a group. The Kurosh rank, denoted $\operatorname{Kr}(\Gamma)$, of a free product decomposition $\Gamma=*_{i \in I} G_{i}$ of $\Gamma$ is defined to be the number of (non-trivial) factors $G_{i}$. By the Kurosh subgroup theorem, any subgroup $H$ of $\Gamma$ inherits a free product decomposition $H=*_{j \in J} H_{j} * F$, where each $H_{j}$ is nontrivial and conjugate to a subgroup of a free factor of $\Gamma$ and $F$ is a free group. The (subgroup) Kurosh rank of $H$ of $\Gamma$ with respect to the above splitting of $\Gamma$, is the sum $|J|+r(F)$, which we again denote by $K r(H)$. The reduced Kurosh rank of $H$ is defined to be $\bar{K} r(H)=\max \{0, K r(H)-1\}$.

Free products also have the Howson property, in the following sense: if $H, K$ are subgroups of $\Gamma$ of finite Kurosh rank, then $H \cap K$ also has finite rank (see [15, Theorem 2.13 (1)] for a proof). In [11], Ivanov proved that if $\Gamma$ is torsion free, then $\bar{K} r(H \cap K) \leq$ $2 \bar{K} r(H) \bar{K} r(K)$. It is shown in [何, that if $\Gamma$ is right-orderable, then the coefficient 2 can be replaced by 1 . 
The problem of finding bounds for the "rank" of the intersection of subgroups in free products and more generally in groups satisfying the Howson property has also been considered in [14, 1, 10, 6, 7, 16, 17, 2].

In this paper, we obtain, under appropriate hypotheses, bounds for the complexity of the intersection of tame subgroups in groups acting on trees with finite edge stabilizers.

Let $G$ be a group acting on a (simplicial) tree $T$ without inversions. A vertex $v$ of $T$ is called $\left(G\right.$-) degenerate if $G_{v}=G_{e}$ for some edge $e$ incident to $v$. The corresponding vertex $[v]_{G}$ of the quotient graph $T / G$ is also called degenerate. Let $H$ be a subgroup of $G$. We denote by $r(T / H)$ the rank of the fundamental group of $T / H$ and by $V_{n d e g}(T / H)$ the set of $H$-non-degenerate vertices of $T / H$. The complexity $C_{T}(H)$ of $H$ with respect to $T$ is defined to be the sum $C_{T}(H)=r(T / H)+\left|V_{\text {ndeg }}(T / H)\right| \in[0, \infty]$, if $H$ contains hyperbolic elements, and 1 otherwise. The reduced complexity of $H$ with respect to $T$, is defined as $\bar{C}_{T}(H)=\max \left\{C_{T}(H)-1,0\right\}$. The subgroup $H$ of $G$ is called tame if either $H$ fixes a vertex, or $H$ contains a hyperbolic element and the quotient graph $T_{H} / H$ is finite, where $T_{H}$ is the unique minimal $H$-invariant subtree of $T$. By [15, Theorem 2.13], if each edge stabilizer is finite, then the intersection of two tame subgroups $H, K$ of $G$ is again tame. In the case where $H \cap K$ fixes a vertex, we obviously have $\bar{C}_{T}(H \cap K) \leq \bar{C}_{T}(H) \cdot \bar{C}_{T}(K)$.

Finitely generated subgroups are examples of tame subgroups. In the case of free products, finite Kurosh rank implies tameness (see Lemma 2.3) and the complexity of a non-trivial subgroup is exactly its Kurosh rank (see section 2 for more details). Our first main result is the following.

Theorem 3.3. Let $G$ be a group acting on a tree $T$ with finite quotient and finite stabilizers of edges and let $H, K$ be tame subgroups of $G$ such that $H \cap K$ does not fix a vertex of $T$.

1. If $T_{H} / H$ and $T_{K} / K$ do not contain degenerate vertices of valence two, then

$$
\bar{C}_{T}(H \cap K) \leq(6 N M+12(M-1) N) \cdot \bar{C}_{T}(H) \cdot \bar{C}_{T}(K),
$$

where $N=\max \left\{\left|G_{x} \cap H K\right|: x \in E T\right\}$ and $M=\max \left\{M_{H}, M_{K}\right\} \leq \max \left\{\left|G_{x}\right|:\right.$ $x \in E T\}$.

2. Suppose $H$ and $K$ satisfy the following property: for each $H$-degenerate (resp. $K$ degenerate) vertex $v$ of $T$, the stabilizer $H_{v}$ (resp. $K_{v}$ ) stabilizes each edge in the star of $v$. Then

$$
\bar{C}_{T}(H \cap K) \leq 6 N \cdot \bar{C}_{T}(H) \cdot \bar{C}_{T}(K) .
$$

In particular, if $H, K$ act freely on the edges of $T$, then

$$
\bar{K}_{T}(H \cap K) \leq 6 N \cdot \bar{K}_{T}(H) \cdot \bar{K}_{T}(K) .
$$


In the special case where both $H$ and $K$ act freely on $T$, the above inequality was proved by Zakharov in [16].

Now let $G=*_{A} G_{i} * F$ be the free product of the amalgamated free product of $G_{i}$ 's with a finite amalgamated subgroup $A$ and $F$, such that $A$ is normal in each $G_{i}$. Following Dicks and Ivanov [6], we define $a_{3}\left(G_{i} / A\right)=\min \left\{|\Gamma|: \Gamma\right.$ is a subgroup of $G_{i} / A$ with $\left.|\Gamma| \geq 3\right\}$ and $\theta\left(G_{i} / A\right)=\left\{\frac{a_{3}\left(G_{i} / A\right)}{a_{3}\left(G_{i} / A\right)-2}\right\} \in[1,3]$, where $\frac{\infty}{\infty-2}:=1$.

We represent $G$ as the fundamental group of a graph of groups $(\mathcal{G}, \Psi)$, where $\Psi$ is the wedge of copies of $[0,1]$ (one copy for each factor $G_{i}$ ) and a bouquet of circles (one for each free generator of $F$ ). To each copy of $[0,1]$ and to the wedge point we associate the group $A$, and to each circle we associate the trivial group. To each of the remaining vertices we associate a factor $G_{i}$. Let $T$ be the corresponding universal tree.

Theorem 3.6. Let $G=*_{A} G_{i} * F$ be the free product of the amalgamated free product of $G_{i}$ 's with a finite amalgamated subgroup $A$ and $F$, such that $A$ is normal in each $G_{i}$. We consider the natural action of $G$ on $T$ defined above. Suppose that $H$ and $K$ are tame subgroups (with respect to $T$ ) of $G$ which act freely on the edges of $T$. Then $H \cap K$ is tame and

$$
\bar{K}_{T}(H \cap K) \leq 2 \cdot \theta \cdot N \cdot \bar{K}_{T}(H) \cdot \bar{K}_{T}(K) \leq 2 \cdot \theta \cdot|A| \cdot \bar{K}_{T}(H) \cdot \bar{K}_{T}(K),
$$

where $\theta=\max \left\{\theta\left(G_{i} / A\right): i \in I\right\}$ and $N=\max \left\{\left|g A g^{-1} \cap H K\right|: g \in G\right\}$.

As an immediate corollary we obtain the main result of [11] mentioned above.

It should be noted that the arguments in the proof of Theorem 3.6, work in a slightly more general setting as well. Thus, with essentially the same proof, we obtain Theorem 3.9 (see also Remark 3.7): If $H, K$ are tame subgroups of a free product $*_{A} G_{i}$ with a finite and normal amalgamated subgroup $A$, then $\bar{C}_{T}(H \cap K) \leq 2 \cdot \theta \cdot|A \cap H K| \cdot \bar{C}_{T}(H) \cdot \bar{C}_{T}(K)$, where $\theta=\max \left\{\theta\left(G_{i} / A\right): i \in I\right\}$ and $T$ is defined as above for $F=1$.

After posting the first version of this paper on the arXiv, the authors learned from A. Zakharov that he, in collaboration with S. Ivanov, had also recently obtained (unpublished) upper bounds for the Kurosh rank of the intersection of free product subgroups in groups acting on trees with finite edge stabilizers.

Acknowledgements. We are grateful to Dimitrios Varsos for many useful discussions and comments. We are also grateful to the anonymous referee for careful reading of the manuscript and pointing out a mistake in an earlier version.

\section{Preliminaries}

To fix our notation, we first recall the definition of a graph. 
Definition 2.1. A graph $X$ consists of a (nonempty) set of vertices $V X$, a set of edges $E X$, a fixed-point free involution ${ }^{-1}: E X \rightarrow E X\left(e \mapsto e^{-1}\right)$ and a map $i: E X \rightarrow V X$. The vertex $i(e)$ is called the initial vertex of the edge $e$. The terminal vertex $t(e)$ of $e$ is defined by $t(e)=i\left(e^{-1}\right)$.

Throughout, let $G$ be a group acting on a (simplicial) tree $T$ (without inversions, i.e. $g e \neq e^{-1}$ for any $g \in G$ and $\left.e \in E X\right)$. By Bass-Serre theory, for which we refer to [5, 13], this is equivalent to saying that $G$ is the fundamental group of the corresponding graph of groups $(\mathcal{G}, T / G)$. If $x \in T$, we denote by $[x]_{G}$ the $G$-orbit of $x$ and by $G_{x}$ its stabilizer. An element $g \in G$ is elliptic if it fixes a vertex of $T$ and hyperbolic otherwise. If $H$ is a subgroup of $G$ containing a hyperbolic element, then there is a unique minimal $H$-invariant subtree $T_{H}$ which is the union of the axes of the hyperbolic elements of $H$.

We recall that a subgroup $H$ of $G$ is called tame if either $H$ fixes a vertex, or $H$ contains a hyperbolic element and the quotient graph $T_{H} / H$ is finite. By [15, Prop. 2.2], the subtree $T_{H}$ is a "core" for the action of $H$ on $T$ in the sense that $r(T / H)+\left|V_{\text {ndeg }}(T / H)\right|=$ $r\left(T_{H} / H\right)+\left|V_{\text {ndeg }}\left(T_{H} / H\right)\right|$, i.e. $C_{T}(H)=C_{T_{H}}(H)$. From this it follows that the complexity of a tame subgroup is finite.

Finitely generated subgroups of $G$ are examples of tame subgroups, since a finitely generated group $\Gamma$ acting by isometries on $T$, either fixes a point of $T$ or else contains a hyperbolic isometry and the quotient graph $T_{\Gamma} / \Gamma$ is finite.

Remark 2.2. We note that if the $G$-stabilizer of each edge is finite and there is a bound on their orders, then any subgroup of $G$ consisting of elliptic elements fixes a vertex of $T$ ([15, Lem. 2.5]).

If we restrict attention to subgroups $H$ of $G$ that act edge-freely on $T$, then the Kurosh $\operatorname{rank} K_{T}(H)$ of $H$ (with respect $T$ ) is defined to be the complexity $C_{T}(H)$ of $H$.

Let $\Gamma=*_{i \in I} G_{i}$ be a free product and $H$ a subgroup of $\Gamma$. By the Kurosh subgroup theorem, $H=*_{i \in I, g_{i}}\left(H \cap g_{i} G_{i} g_{i}^{-1}\right) * F$, where for each $i, g_{i}$ ranges over a set of double coset representatives in $G_{i} \backslash \Gamma / H$ and $F$ is a free group intersecting each conjugate $g G_{i} g^{-1}$ trivially. The (subgroup) Kurosh rank of $H$ with respect the above free product decomposition of $\Gamma$, denoted by $K r(H)$, is the sum $|\Lambda|+\operatorname{rank}(F)$, where $|\Lambda|$ is the number of all non-trivial factors $H \cap g_{i} G_{i} g_{i}^{-1}$. Note that the Kurosh rank of $\Gamma$ is the number of non-trivial factors $G_{i}$.

It is not difficult to verify that the numbers $|\Lambda| \operatorname{rank}(F)$ depend only on $H$ and the given free product decomposition of $\Gamma$. In fact, if $T$ is any $\Gamma$-tree corresponding to the given decomposition of $\Gamma$, then the Kurosh rank of $H$ with respect to $\Gamma=*_{i \in I} G_{i}$ is equal to the Kurosh rank $K_{T}(H)$ of the associated free product decomposition of $H$ coming from the action of $H$ on $T$. Thus, if $H$ is non-trivial, then $K r(H)=K_{T}(H)=C_{T}(H)$. 
Lemma 2.3. Let $G$ be a group acting on a tree $T$ and $H$ a subgroup of $G$ that act edgefreely on $T$. If $K_{T}(H)<\infty$, then $H$ is tame.

Proof. It suffices to consider the case when $H$ contains a hyperbolic element. Let $\pi$ : $T \rightarrow T / H$ be the natural projection given by $\pi(x)=[x]_{H}$. Since $K_{T}(H)<\infty$, there are finitely many vertices $v_{1}, \ldots, v_{n}$ of $T / H$ with non-trivial group and finitely many edges $e_{1}, \ldots, e_{m}$ of $T / H$ such that $X=T / H \backslash\left\{e_{1}, \ldots, e_{m}\right\}$ is a maximal tree of $T / H$. Let $Y$ be the finite subgraph of $T / H$ consisting of $\left\{e_{1}, \ldots, e_{m}\right\}$ and all geodesics in $X$ between endpoints of the $e_{i}^{\prime} s$ and $v_{1}, \ldots, v_{n}$. We claim that $\pi^{-1}(Y)$ is connected. To see this, let $p=x_{1} \cdots x_{k}$ be a reduced path connecting vertices of $\pi^{-1}(Y)$ such that no edge of $p$ lies in $\pi^{-1}(Y)$. Then $\pi(p)$ is contained in the complement $T / H \backslash Y$ of $Y$. Since $Y$ contains the edges $e_{1}, \ldots, e_{m}$, each component $C$ of $T / H \backslash Y$ is a tree, and it is not difficult to see that $C$ intersects $T / H \backslash Y$ in only one vertex. It follows that there is an index $i$ such that $\pi\left(x_{i}\right)=\pi\left(x_{i+1}\right)^{-1}$. This means that $h x_{i}=x_{i+1}^{-1}$ for some $h \in H$ and hence $h$ fixes the initial vertex $v$ of $x_{i}$. From the construction of $Y, v$ is degenerate and therefore $h=1$, which contradicts the choice of $p$.

Thus, $\pi^{-1}(Y)$ is a connected $H$-invariant subgraph of $T$. It follows that $T_{H} \subseteq \pi^{-1}(Y)$. We conclude that $T_{H} / H$ is finite, being a subgraph of $Y$.

\section{Proofs of the main results}

Let $Y$ be a graph and $v$ a vertex of $Y$. The star of $v$, denoted $\operatorname{Star}_{Y}(v)$, is the set of edges of $Y$ with initial vertex $v$, i.e. $\operatorname{Star}_{Y}(v)=\{e \in E Y \mid i(e)=v\}$. The valence or degree of $v$ in $Y$, denoted $\operatorname{deg}_{Y}(v)$, is the number of edges in the star of $v$.

Lemma 3.1. Let $G$ be a group acting on a tree $T$, let $H$ be a tame subgroup of $G$ containing hyperbolic elements and let $\tilde{X}$ be the graph obtained from $X=T_{H} / H$ by attaching a loop at each $H$-non-degenerate vertex. Then

$$
\bar{C}_{T}(H)=\bar{r}(\widetilde{X})=\frac{1}{2} \sum\left(\operatorname{deg}_{\widetilde{X}}\left([v]_{H}\right)-2\right)
$$

where the sum is taken over all vertices $[v]_{H}$ of $\tilde{X}$.

Proof. The reduced rank of a graph is equal to the number of its (geometric-oriented) edges minus the number of its vertices. The minimality of $T_{H}$ implies that each vertex of $X$ of valence one is $H$-non-degenerate. Therefore, every vertex of $\widetilde{X}$ has valence at least two. Now an easy calculation shows that the $\operatorname{sum} \sum\left(\operatorname{deg}_{\widetilde{X}}\left([v]_{H}\right)-2\right)$, over all vertices $[v]_{H}$ of $\tilde{X}$, is equal to $2 \bar{r}(\widetilde{X})$. By construction of $\widetilde{X}$, we have $\bar{r}(\widetilde{X})=\bar{C}_{T}(H)$ which completes the proof. 
Lemma 3.2. Let $G$ be a group acting on a tree $T$ and let $A$ and $B$ be subgroups of $G$ such that $A \subseteq B$. Suppose that $A$ and $B$ contain hyperbolic elements and that $v$ is a vertex of $T_{B}$. We consider the graph map $\pi_{B}: T_{A} / A \longrightarrow T_{B} / B$ given by $\pi\left([x]_{A}\right)=[x]_{B}$.

1. $\left|\operatorname{Star}\left([v]_{A}\right)\right| \leq\left|G_{v} \cap B\right| \cdot\left|\operatorname{Star}\left([v]_{B}\right)\right|$ (provided that they are finite).

2. If, moreover, $B_{v}$ is B-degenerate and stabilizes each edge in $\operatorname{Star}_{T_{B}}(v)$, then the restriction $\pi_{B}: \operatorname{Star}\left([v]_{A}\right) \longrightarrow \operatorname{Star}\left([v]_{B}\right)$ is an embedding.

Proof. Suppose that $\left[e_{1}\right]_{A}$ and $\left[e_{2}\right]_{A}$ are two edges in the star of $[v]_{A}$ with $\pi\left(\left[e_{1}\right]_{A}\right)=$ $\pi\left(\left[e_{2}\right]_{A}\right)$. Then there are $a_{1}, a_{2} \in A$ and $b \in B$ such that $i\left(e_{1}\right)=a_{1} v, i\left(e_{2}\right)=a_{2} v$ and $e_{1}=b e_{2}$. It follows that $i\left(e_{1}\right)=b i\left(e_{2}\right)$ and thus $a_{1} v=b a_{2} v$. Hence $a_{1}^{-1} b a_{2} \in G_{v} \cap B=B_{v}$. Now, if $[x]_{A}$ is an edge in the star of $[v]_{A}$ with $\pi\left([x]_{A}\right)=\pi\left(\left[e_{1}\right]_{A}\right)=\pi\left(\left[e_{2}\right]_{A}\right)$, then as before $i(x)=a_{x} v$ and $x=b_{x} e_{2}$ for some $a_{x} \in A$ and $b_{x} \in B$. If we assume further that $a_{1}^{-1} b a_{2}=$ $a_{x}^{-1} b_{x} a_{2}$, then $a_{1}^{-1} b=a_{x}^{-1} b_{x}$ and so $\left[e_{1}\right]_{A}=\left[b e_{2}\right]_{A}=\left[a_{1} a_{x}^{-1} b_{x} e_{2}\right]_{A}=\left[b_{x} e_{2}\right]_{A}=[x]_{A}$. This means that each fiber of the restriction (on stars) has at most $\left|G_{v} \cap B\right|$ elements, and the first assertion follows.

Now, if $B_{v}$ stabilizes each edge in $\operatorname{Star}_{T_{B}}(v)$, then $a_{1}^{-1} b a_{2}$ stabilizes $a_{2}^{-1} e_{2}$ and therefore $\left[e_{1}\right]_{A}=\left[b e_{2}\right]_{A}=\left[a_{1} a_{2}^{-1} e_{2}\right]_{A}=\left[e_{2}\right]_{A}$.

In view of this lemma, we define $M_{B}:=\max \left\{\left|G_{v} \cap B\right|: v\right.$ is a B-degenerate vertex of $\left.\mathrm{T}\right\}$.

The following is our first main result.

Theorem 3.3. Let $G$ be a group acting on a tree $T$ with finite quotient and finite stabilizers of edges and let $H, K$ be tame subgroups of $G$ such that $H \cap K$ does not fix a vertex of $T$.

1. If $T_{H} / H$ and $T_{K} / K$ do not contain degenerate vertices of valence two, then

$$
\bar{C}_{T}(H \cap K) \leq(6 N M+12(M-1) N) \cdot \bar{C}_{T}(H) \cdot \bar{C}_{T}(K),
$$

where $N=\max \left\{\left|G_{x} \cap H K\right|: x \in E T\right\}$ and $M=\max \left\{M_{H}, M_{K}\right\} \leq \max \left\{\left|G_{x}\right|:\right.$ $x \in E T\}$.

2. Suppose $H$ and $K$ satisfy the following property: for each $H$-degenerate (resp. $K$ degenerate) vertex $v$ of $T$, the stabilizer $H_{v}$ (resp. $K_{v}$ ) stabilizes each edge in the star of $v$. Then

$$
\bar{C}_{T}(H \cap K) \leq 6 N \cdot \bar{C}_{T}(H) \cdot \bar{C}_{T}(K) .
$$

In particular, if $H, K$ act freely on the edges of $T$, then

$$
\bar{K}_{T}(H \cap K) \leq 6 N \cdot \bar{K}_{T}(H) \cdot \bar{K}_{T}(K) .
$$

Proof. Since $H \cap K$ does not fix a vertex, it follows from Remark 2.2 that $H \cap K, H$ and $K$ contain hyperbolic elements. Let $T_{H \cap K}, T_{H}, T_{K}$ be the minimal subtrees of $T$ 
invariant under $H \cap K, H, K$, respectively. Let $\pi_{H}: T_{H \cap K} / H \cap K \longrightarrow T_{H} / H$ and $\pi_{K}: T_{H \cap K} / H \cap K \longrightarrow T_{K} / K$ be the natural projections (defined as in Lemma 3.2). We consider the map $\pi=\left(\pi_{H}, \pi_{K}\right): T_{H \cap K} / H \cap K \longrightarrow T_{H} / H \times T_{K} / K$ given by $\pi\left([x]_{H \cap K}\right)=$ $\left([x]_{H},[x]_{K}\right)$. By [3, Proposition 8.7], each fiber $\pi^{-1}\left([x]_{H},[x]_{K}\right)$, where $x$ is an edge or a vertex, has exactly $\left|H_{x} \backslash G_{x} \cap H K / K_{x}\right|$ elements. It follows that for each edge $x$ the fiber $\pi^{-1}\left([x]_{H},[x]_{K}\right)$ has at most $N$ elements.

For convenience we simplify notation by setting $X=T_{H \cap K} / H \cap K, Y=T_{H} / H$ and $Z=T_{K} / K$. As in Lemma 3.1, we construct graphs $\widetilde{X}, \widetilde{Y}$ and $\widetilde{Z}$, by attaching a loop at each non-degenerate vertex of $X, Y$ and $Z$, respectively.

1) By Lemma 3.1, it suffices to show that

$$
\sum_{V \widetilde{X}}\left(\operatorname{deg}_{\widetilde{X}}\left([v]_{H \cap K}\right)-2\right) \leq(3 N M+6 N(M-1)) \sum_{V \widetilde{Y}}\left(\operatorname{deg}_{\widetilde{Y}}\left([v]_{H}\right)-2\right) \cdot \sum_{V \widetilde{Z}}\left(\operatorname{deg}_{\widetilde{Z}}\left([v]_{K}\right)-2\right) .
$$

For any pair of vertices $(a, b) \in Y \times Z$, we will show that

$$
\sum_{v \in \pi^{-1}(a, b)}\left(\operatorname{deg}_{\widetilde{X}}(v)-2\right) \leq(3 N M+6 N(M-1)) \cdot\left(\operatorname{deg}_{\widetilde{Y}}(a)-2\right) \cdot\left(\operatorname{deg}_{\widetilde{Z}}(b)-2\right)
$$

from which (1) follows. The rest of the proof follows similar arguments to those given in [4], [6] and [11]. Let $\left\{v_{1}, \ldots, v_{n}\right\}$ be the vertices of $\pi^{-1}(a, b)$. Since the fiber of any edge of $Y \times Z$ contains at most $N$ edges, we have

$$
\sum_{i=1}^{n} \operatorname{deg}_{X}\left(v_{i}\right) \leq N \cdot \operatorname{deg}_{Y}(a) \cdot \operatorname{deg}_{Z}(b) .
$$

We consider three cases depending on whether or not $a$ and $b$ are degenerate.

Case 1. Suppose that $a$ is $H$-non-degenerate and $b$ is $K$-non-degenerate. Then $\operatorname{deg}_{Y}(a)=$ $\operatorname{deg}_{\widetilde{Y}}(a)-2, \operatorname{deg}_{Z}(b)=\operatorname{deg}_{\widetilde{Z}}(b)-2$ while $\operatorname{deg}_{X}\left(v_{i}\right)$ is equal to $\operatorname{deg}_{\widetilde{X}}\left(v_{i}\right)-2 \operatorname{or} \operatorname{deg}_{\widetilde{X}}\left(v_{i}\right)$. Hence

$\sum_{i=1}^{n}\left(\operatorname{deg}_{\widetilde{X}}\left(v_{i}\right)-2\right) \leq \sum_{i=1}^{n} \operatorname{deg}_{X}\left(v_{i}\right) \leq N \cdot \operatorname{deg}_{Y}(a) \cdot \operatorname{deg}_{Z}(b)=N \cdot\left(\operatorname{deg}_{\widetilde{Y}}(a)-2\right) \cdot\left(\operatorname{deg}_{\widetilde{Z}}(b)-2\right)$.

Case 2. Exactly one of $a, b$, say $b$, is degenerate. Then each $v_{i}$ is $(H \cap K)$-degenerate as well, and thus $\operatorname{deg}_{Y}(a)=\operatorname{deg}_{\widetilde{Y}}(a)-2, \operatorname{deg}_{X}\left(v_{i}\right)=\operatorname{deg}_{\widetilde{X}}\left(v_{i}\right)$ and $\operatorname{deg}_{\widetilde{Z}}(b)=\operatorname{deg}_{Z}(b)>2$. Also, by Lemma 3.2, for each $i$ we have $\operatorname{deg}_{X}\left(v_{i}\right) \leq M \operatorname{deg}_{Z}(b)$.

If $n \leq N \cdot \operatorname{deg}_{Y}(a)$, then

$$
\begin{aligned}
\sum_{i=1}^{n}\left(\operatorname{deg}_{\widetilde{X}}\left(v_{i}\right)-2\right) & =\sum_{i=1}^{n}\left(\operatorname{deg}_{X}\left(v_{i}\right)-2\right) \leq n \cdot\left(M \operatorname{deg}_{Z}(b)-2\right) \leq N \cdot \operatorname{deg}_{Y}(a)\left(M \operatorname{deg}_{Z}(b)-2\right) \\
& \leq N \cdot \operatorname{deg}_{Y}(a)\left(M\left(\operatorname{deg}_{Z}(b)-2\right)+2(M-1)\right) \\
& =N M\left(\operatorname{deg}_{\widetilde{Y}}(a)-2\right)\left(\operatorname{deg}_{\widetilde{Z}}(b)-2\right)+2 N(M-1)\left(\operatorname{deg}_{\widetilde{Y}}(a)-2\right)(4) \\
& \leq(N M+2 N(M-1)) \cdot\left(\operatorname{deg}_{\widetilde{Y}}(a)-2\right) \cdot\left(\operatorname{deg}_{\widetilde{Z}}(b)-2\right)
\end{aligned}
$$


where the last inequality follows because $\operatorname{deg}_{\widetilde{Z}}(b)>2$.

On the other hand, if $n \geq N \cdot \operatorname{deg}_{Y}(a)$, then

$$
\begin{aligned}
\sum_{i=1}^{n}\left(\operatorname{deg}_{\widetilde{X}}\left(v_{i}\right)-2\right) & =\sum_{i=1}^{n} \operatorname{deg}_{X}\left(v_{i}\right)-2 n \leq N \cdot \operatorname{deg}_{Y}(a) \cdot \operatorname{deg}_{Z}(b)-2 N \cdot \operatorname{deg}_{Y}(a) \\
& =N \operatorname{deg}_{Y}(a) \cdot\left(\operatorname{deg}_{Z}(b)-2\right)=N\left(\operatorname{deg}_{\widetilde{Y}}(a)-2\right)\left(\operatorname{deg}_{\widetilde{Z}}(b)-2\right) .
\end{aligned}
$$

Case 3. Finally, suppose that $a, b$ are degenerate in $Y, Z$, respectively. Then each vertex $v_{i}$ is $(H \cap K)$-degenerate as well and $\operatorname{deg}_{\widetilde{Y}}(a)=\operatorname{deg}_{Y}(a)>2, \operatorname{deg}_{\widetilde{X}}\left(v_{i}\right)=\operatorname{deg}_{X}\left(v_{i}\right)$, $\operatorname{deg}_{\widetilde{Z}}(b)=\operatorname{deg}_{Z}(b)>2$. Moreover, by Lemma 3.2, $\operatorname{deg}_{X}\left(v_{i}\right) \leq \min \left\{M \operatorname{deg}_{Y}(a), M \operatorname{deg}_{Z}(b)\right\}$. Suppose that $\operatorname{deg}_{Z}(b)=\min \left\{\operatorname{deg}_{Y}(a), \operatorname{deg}_{Z}(b)\right\}$ and hence $\operatorname{deg}_{Y}(a)=\max \left\{\operatorname{deg}_{Y}(a), \operatorname{deg}_{Z}(b)\right\}$ (the other case is handled in the same way).

If $n \leq N \cdot \operatorname{deg}_{Y}(a)$, then

$\sum_{i=1}^{n}\left(\operatorname{deg}_{\widetilde{X}}\left(v_{i}\right)-2\right)=\sum_{i=1}^{n}\left(\operatorname{deg}_{X}\left(v_{i}\right)-2\right) \leq n \cdot\left(M \operatorname{deg}_{Z}(b)-2\right) \leq N \cdot \operatorname{deg}_{Y}(a) \cdot\left(M \operatorname{deg}_{Z}(b)-2\right)$.

On the other hand, if $n \geq N \cdot \operatorname{deg}_{Y}(a)$, then

$\sum_{i=1}^{n}\left(\operatorname{deg}_{\widetilde{X}}\left(v_{i}\right)-2\right)=\sum_{i=1}^{n} \operatorname{deg}_{X}\left(v_{i}\right)-2 n \leq N \cdot \operatorname{deg}_{Y}(a) \cdot \operatorname{deg}_{Z}(b)-2 N \cdot \operatorname{deg}_{Y}(a) \leq N \cdot \operatorname{deg}_{Y}(a) \cdot\left(\operatorname{deg}_{Z}(b)-2\right)$.

Thus, in each case we have

$$
\sum_{i=1}^{n}\left(\operatorname{deg}_{\tilde{X}}\left(v_{i}\right)-2\right) \leq N \cdot \operatorname{deg}_{Y}(a) \cdot\left(M \operatorname{deg}_{Z}(b)-2\right) .
$$

Since $\operatorname{deg}_{Y}(a) \geq 3$, or equivalently, $\operatorname{deg}_{Y}(a) \leq 3\left(\operatorname{deg}_{Y}(a)-2\right)$, it follows that

$$
\begin{aligned}
\sum_{i=1}^{n}\left(\operatorname{deg}_{\widetilde{X}}\left(v_{i}\right)-2\right) & \leq 3 N \cdot\left(\operatorname{deg}_{Y}(a)-2\right) \cdot\left(M \operatorname{deg}_{Z}(b)-2\right) \\
& \leq 3 N \cdot\left(\operatorname{deg}_{Y}(a)-2\right) \cdot\left(M\left(\operatorname{deg}_{Z}(b)-2\right)+2(M-1)\right) \\
& =3 N M \cdot\left(\operatorname{deg}_{Y}(a)-2\right) \cdot\left(\operatorname{deg}_{Z}(b)-2\right)+6 N(M-1) \cdot\left(\operatorname{deg}_{Y}(a)-2\right) \\
& \leq(3 N M+6 N(M-1)) \cdot\left(\operatorname{deg}_{\widetilde{Y}}(a)-2\right) \cdot\left(\operatorname{deg}_{\widetilde{Z}}(b)-2\right) .
\end{aligned}
$$

This completes the proof of part 1) of the theorem.

2) To prove the second part, again by Lemma 3.1, it suffices to show that

$$
\sum_{v \in \pi^{-1}(a, b)}\left(\operatorname{deg}_{\widetilde{X}}(v)-2\right) \leq 3 N\left(\operatorname{deg}_{\widetilde{Y}}(a)-2\right) \cdot\left(\operatorname{deg}_{\widetilde{Z}}(b)-2\right)
$$

for each pair of vertices $(a, b) \in Y \times Z$. Proceeding exactly as before, we distinguish three cases. In Case 1, where both $a$ and $b$ are non-degenerate, we get the same inequality. In Cases 2 and 3, by Lemma 3.2 (2), we can now use 1 instead of $M$. Thus in Cases 2 and 3 , we obtain respectively (from 4 国 and 7 ) the inequalities

$$
\sum_{i=1}^{n}\left(\operatorname{deg}_{\widetilde{X}}\left(v_{i}\right)-2\right) \leq N\left(\operatorname{deg}_{\widetilde{Y}}(a)-2\right) \cdot\left(\operatorname{deg}_{\widetilde{Z}}(b)-2\right)
$$


and

$$
\sum_{i=1}^{n}\left(\operatorname{deg}_{\widetilde{X}}\left(v_{i}\right)-2\right) \leq 3 N\left(\operatorname{deg}_{\widetilde{Y}}(a)-2\right) \cdot\left(\operatorname{deg}_{\widetilde{Z}}(b)-2\right) .
$$

It remains only to consider the case when both $a$ and $b$ are degenerate (in which case we are in Case 3) and $\operatorname{deg}_{Y}(a)=2$, where $a$ is the vertex of maximal degree. If $\operatorname{deg}_{Y}(a)=2$, then $\operatorname{deg}_{Z}(b)=2$ too, and inequality 8 follows since, by Lemma $3.2(2), \operatorname{deg}_{\widetilde{X}}\left(v_{i}\right)=\operatorname{deg}_{X}\left(v_{i}\right) \leq$ $\min \left\{\operatorname{deg}_{Y}(a), \operatorname{deg}_{Z}(b)\right\}$ for each $i$.

Corollary 3.4. (10, Theorem 1]) Let $G$ be a group acting on a tree $T$ with finite quotient and finite stabilizers of edges and let $H, K$ be finitely generated subgroups of $G$ which intersect trivially each vertex stabilizer (and hence they are free groups). Then $H \cap K$ is finitely generated and

$$
\bar{r}(H \cap K) \leq 6 N \cdot \bar{r}(H) \cdot \bar{r}(K),
$$

where $N=\max \left\{\left|G_{x} \cap H K\right|: x \in E T\right\}$.

Corollary 3.5. Let $G$ be a group acting on a tree $T$ with finite quotient, finite stabilizers of edges and infinite vertex stabilizers. If $H$ and $K$ are subgroups of finite index in $G$, then

$$
\bar{C}_{T}(H \cap K) \leq 2 N \cdot \bar{C}_{T}(H) \cdot \bar{C}_{T}(K) .
$$

Proof. If the $G$-stabilizer of every vertex is infinite and both $H$ and $K$ are of finite index in $G$, then each vertex stabilizer is also infinite under the action of $H$ or $K$ (being of finite index in the corresponding $G$-stabilizer) and thus Cases 2 and 3 do not occur.

Following [6], given a group $G$, we define $a_{3}(G)=\min \{|\Gamma|: \Gamma$ is a subgroup of $G$ with $|\Gamma| \geq$ $3\}$ and $\theta(G)=\left\{\frac{a_{3}(G)}{a_{3}(G)-2}\right\} \in[1,3]$, where $\frac{\infty}{\infty-2}:=1$.

In the sequel, we prove that if $H, K$ act freely on the edges, then the coefficient 6 in the above theorem can be replaced by a number $2 \theta$, where $\theta \in[1,3]$, by imposing some extra hypotheses on the structure of $G$.

Let $G_{i}, i \in I$, be a family of groups together with a group $A$, let $\phi_{i}: A \longrightarrow G_{i}$ be a family of monomorphisms and let $*_{A} G_{i}$ be the amalgamated free product of $G_{i}$ 's with amalgamated subgroup $A$ (with respect to $\phi_{i}$ ). We can think of each $\phi_{i}$ as an inclusion. Let $F$ be a free group and let $G=*_{A} G_{i} * F$ be the free product of $F$ and $*_{A} G_{i}$. We construct a graph of groups $(\mathcal{G}, \Psi)$ with fundamental group $G$ as follows. The graph $\Psi$ consists of a wedge of open edges $e_{i}=\left[u_{0}, u_{i}\right], i \in I$ (i.e. one for each factor $G_{i}$ and distinct endpoints $u_{0}$ and $\left.u_{i}, i \in I, 0 \notin I\right)$, together with a wedge of loops $l_{j}$, one for each free generator of $F$, attached at a vertex $u_{0}$ with vertex group $A$. To each edge $e_{i}$ we associate the group $A$, to each loop $l_{j}$ we associate the trivial group and to each vertex $u_{i}$ we associate the group $G_{i}$. We denote by $T$ the corresponding universal tree. 
Theorem 3.6. Let $G=*_{A} G_{i} * F$ be the free product of the amalgamated free product of $G_{i}$ 's with a finite amalgamated subgroup $A$ and $F$, such that $A$ is normal in each $G_{i}$. We consider the natural action of $G$ on $T$ defined above. Suppose that $H$ and $K$ are tame subgroups (with respect to $T$ ) of $G$ which act freely on the edges of $T$. Then $H \cap K$ is tame and

$$
\bar{K}_{T}(H \cap K) \leq 2 \cdot \theta \cdot N \cdot \bar{K}_{T}(H) \cdot \bar{K}_{T}(K) \leq 2 \cdot \theta \cdot|A| \cdot \bar{K}_{T}(H) \cdot \bar{K}_{T}(K),
$$

where $\theta=\max \left\{\theta\left(G_{i} / A\right): i \in I\right\}$ and $N=\max \left\{\left|g A g^{-1} \cap H K\right|: g \in G\right\}$.

Proof. We proceed as in the proof of Theorem 3.3. With the notation of that proof, we have to prove that

$$
\sum_{i=1}^{n}\left(\operatorname{deg}_{\widetilde{X}}\left(v_{i}\right)-2\right) \leq \theta \cdot N \cdot\left(\operatorname{deg}_{\widetilde{Y}}(a)-2\right) \cdot\left(\operatorname{deg}_{\widetilde{Z}}(b)-2\right)
$$

for any pair of vertices $(a, b) \in Y \times Z$ (recall that $\left\{v_{1}, \ldots, v_{n}\right\}$ denotes the vertices of $\left.\pi^{-1}(a, b)\right)$. Since $H, K$ act freely on the edges of $T$, it follows, by Lemma 3.2 (2), that we can use 1 instead of $M$. Suppose first that at least one of $a$ and $b$ is non-degenerate. Then the arguments of Cases 1, 2 of the proof of Theorem 3.3 apply to show that

$$
\sum_{i=1}^{n}\left(\operatorname{deg}_{\widetilde{X}}\left(v_{i}\right)-2\right) \leq N \cdot\left(\operatorname{deg}_{\widetilde{Y}}(a)-2\right) \cdot\left(\operatorname{deg}_{\widetilde{Z}}(b)-2\right)
$$

and inequality 11 holds. Thus it suffices to consider the case where $a$ is $H$-degenerate and $b$ is $K$-degenerate (i.e. Case 3 in the proof of Theorem 3.3). In this case we have $\operatorname{deg}_{Y}(a)=\operatorname{deg}_{\widetilde{Y}}(a), \operatorname{deg}_{Z}(b)=\operatorname{deg}_{\widetilde{Z}}(b)$ and $\operatorname{deg}_{X}\left(v_{i}\right)=\operatorname{deg}_{\widetilde{X}}\left(v_{i}\right)$, while by Lemma 3.2 $(2), \operatorname{deg}_{X}\left(v_{i}\right) \leq \min \left\{\operatorname{deg}_{Y}(a), \operatorname{deg}_{Z}(b)\right\}$. For each $i \in\{1, \ldots, n\}$, choose a vertex, $w_{i}$, of $T$, so that $\left[w_{i}\right]_{H \cap K}=v_{i} \in \pi^{-1}(a, b)$. Note that all $w_{1}, \ldots, w_{n}$ lie in the same $G$-orbit. There are two subcases to consider.

(i) $w_{i}$ and $u_{0}$ are in the same $G$-orbit, i.e. $w_{i}=g_{i} u_{0}$ for some $g_{i} \in G$. Notice that $H_{w_{i}}=K_{w_{i}}=g_{i} A g_{i}^{-1} \cap H=g_{i} A g_{i}^{-1} \cap K=1$. If one of the vertices $a$ or $b$ has valence 2 , then (each) $v_{i}$ has valence 2 as well and inequality 11 is obvious. If both $a$ and $b$ have valence at least 3 , then

$$
\begin{aligned}
\sum_{i=1}^{n}\left(\operatorname{deg}_{\widetilde{X}}\left(v_{i}\right)-2\right) & \leq n \cdot\left(\operatorname{deg}_{\widetilde{Y}}(a)-2\right) \leq\left|H_{w_{i}} \backslash G_{w_{i}} \cap H K / K_{w_{i}}\right|\left(\operatorname{deg}_{\widetilde{Y}}(a)-2\right) \cdot(3-2) \\
& \leq N \cdot\left(\operatorname{deg}_{\widetilde{Y}}(a)-2\right) \cdot\left(\operatorname{deg}_{\widetilde{Z}}(b)-2\right) \leq \theta(G) \cdot N \cdot\left(\operatorname{deg}_{\widetilde{Y}}(a)-2\right) \cdot\left(\operatorname{deg}_{\widetilde{Z}}(b)-2\right) .
\end{aligned}
$$

(ii) $w_{i}$ and $u_{j}$ are in the same $G$-orbit for some $j \in I$, i.e. there exists $g_{i} \in G$ such that $w_{i}=g_{i} u_{j}$. As before, we may assume that both $a$ and $b$ have valence at least 3 .

If $n \leq N$, then

$$
\sum_{i=1}^{n}\left(\operatorname{deg}_{\widetilde{X}}\left(v_{i}\right)-2\right) \leq N \cdot\left(\operatorname{deg}_{\widetilde{Y}}(a)-2\right) \cdot(3-2) \leq N \cdot\left(\operatorname{deg}_{\widetilde{Y}}(a)-2\right) \cdot\left(\operatorname{deg}_{\widetilde{Z}}(b)-2\right) .
$$


Suppose now that $n>N$. Let $\mathcal{R}=\left\{g_{\lambda}\right\}_{\lambda \in \Lambda}$ be a set of representatives of left cosets of $A$ in $G_{j}$. From the construction of $X=T / G$, the stars of different vertices of $\pi^{-1}(a, b)$ are disjoint, while each edge in the star of $\left[g_{i} u_{j}\right]_{\Gamma}$, where $\Gamma=H \cap K, H$ or $K$, is of the form $\left[x e_{j}\right]_{\Gamma}$ and its terminal vertex is $\left[g_{i} u_{j}\right]_{\Gamma}$. It follows that there is $\gamma \in \Gamma$ such that $\gamma x u_{j}=g_{i} u_{j}$ and thus $g_{i}^{-1} \gamma x \in G_{j}$. If we write $g_{i}^{-1} \gamma x$ as $g_{\lambda(x)} a$, where $g_{\lambda(x)} \in \mathcal{R}$ and $a \in A$, then $\left[x e_{j}\right]_{\Gamma}=\left[\gamma^{-1} g_{i} g_{\lambda(x)} a e_{j}\right]_{\Gamma}=\left[g_{i} g_{\lambda(x)} a e_{j}\right]_{\Gamma}=\left[g_{i} g_{\lambda(x)} e_{j}\right]_{\Gamma}$. It follows that there exists a subset $\mathcal{R}_{\Gamma}^{i}$ of $\mathcal{R}$ such that $\operatorname{Star}\left(\left[w_{i}\right]_{\Gamma}\right)=\left\{\left[g_{i} g_{\lambda} e_{j}\right]_{\Gamma}: g_{\lambda} \in \mathcal{R}_{\Gamma}^{i}\right\}$ and $\left|\mathcal{R}_{\Gamma}^{i}\right|=\left|\operatorname{Star}\left(\left[w_{i}\right]_{\Gamma}\right)\right|$. In particular, $\left|\mathcal{R}_{H}^{i}\right|=\left|\operatorname{Star}_{Y}(a)\right|$ and $\left|\mathcal{R}_{K}^{i}\right|=\left|\operatorname{Star}_{Z}(b)\right|$.

Fix $i \in\{1, \ldots, n\}$. For any $k \in\{1, \ldots, n\}$, let $C_{k}$ be the subset of $\mathcal{R}_{H} \times \mathcal{R}_{K}:=$ $\mathcal{R}_{H}^{i} \times \mathcal{R}_{K}^{i}$ consisting of all pairs $\left(g_{\lambda}, g_{\mu}\right)$ such that $\left(\left[g_{i} g_{\lambda} e_{j}\right]_{H},\left[g_{i} g_{\mu} e_{j}\right]_{K}\right)$ is the image under $\pi$ of some edge $\left[x e_{j}\right]_{H \cap K}$ in the star of $\left[g_{k} u_{j}\right]_{H \cap K}=v_{k}$ in $X$. Let $\phi: G_{j} \rightarrow G_{j} / A$ denote the natural epimorphism. Note that the restriction of $\phi$ on $\mathcal{R}$ is a bijection.

We will show that $\phi\left(C_{k}\right)=\left\{\left(\phi\left(g_{\lambda}\right), \phi\left(g_{\mu}\right)\right):\left(g_{\lambda}, g_{\mu}\right) \in C_{k}\right\}$ is a single-quotient subset of $\phi\left(\mathcal{R}_{H}\right) \times \phi\left(\mathcal{R}_{K}\right)$, in the terminology of [6], i.e. that the product $\phi\left(g_{\lambda}\right) \cdot \phi\left(g_{\mu}\right)^{-1}$ is constant for all pairs $\left(g_{\lambda}, g_{\mu}\right) \in C_{k}$. Suppose that $y_{1}$ and $y_{2}$ are edges in the star of $v_{k}$ and that $\pi\left(y_{t}\right)=\left(\left[g_{i} g_{\lambda(t)} e_{j}\right]_{H},\left[g_{i} g_{\mu(t)} e_{j}\right]_{K}\right), t=1,2$. We want to show that $\phi\left(g_{\lambda(1)}\right) \cdot \phi\left(g_{\mu(1)}\right)^{-1}=$ $\phi\left(g_{\lambda(2)}\right) \cdot \phi\left(g_{\mu(2)}\right)^{-1}$. From the above analysis, we can write $y_{t}=\left[g_{k} g_{s(t)} e_{j}\right]_{H \cap K}$ for some $g_{s(t)} \in \mathcal{R}_{H \cap K}^{k}, t=1,2$, and thus $\pi\left(y_{t}\right)=\left(\left[g_{k} g_{s(t)} e_{j}\right]_{H},\left[g_{k} g_{s(t)} e_{j}\right]_{K}\right)$. It follows that $\left(\left[g_{k} g_{s(1)} e_{j}\right]_{H},\left[g_{k} g_{s(1)} e_{j}\right]_{K}\right)=\left(\left[g_{i} g_{\lambda(1)} e_{j}\right]_{H},\left[g_{i} g_{\mu(1)} e_{j}\right]_{K}\right)$, and that $\left(\left[g_{k} g_{s(2)} e_{j}\right]_{H},\left[g_{k} g_{s(2)} e_{j}\right]_{K}\right)=$ $\left(\left[g_{i} g_{\lambda(2)} e_{j}\right]_{H},\left[g_{i} g_{\mu(2)} e_{j}\right]_{K}\right)$. Hence there are $h_{1}, h_{2} \in H, k_{1}, k_{2} \in K$ and $a_{1}, a_{2}, a_{3}, a_{4} \in A$ such that

$$
\begin{array}{ll}
g_{k} g_{s(1)}=h_{1} g_{i} g_{\lambda(1)} a_{1}, & g_{k} g_{s(2)}=h_{2} g_{i} g_{\lambda(2)} a_{3} \\
g_{k} g_{s(1)}=k_{1} g_{i} g_{\mu(1)} a_{2}, & g_{k} g_{s(2)}=k_{2} g_{i} g_{\mu(2)} a_{4}
\end{array}
$$

By normality of $A$ in $G_{j}$, the stabilizer of any edge in the star of $w_{i}$ is equal to $g_{i} A g_{i}^{-1}$. Therefore our assumption that $w_{i}$ is $H, K$ degenerate implies that $H \cap G_{w_{i}}=H \cap g_{i} A g_{i}^{-1}$ and $K \cap G_{w_{i}}=K \cap g_{i} A g_{i}^{-1}$. Now, from the first two equalities above we deduce that

$$
h_{2}^{-1} h_{1}=g_{i} g_{\lambda(2)} a_{3} g_{s(2)}^{-1} g_{s(1)} a_{1}^{-1} g_{\lambda(1)}^{-1} g_{i}^{-1} \in H \cap g_{i} G_{j} g_{i}^{-1}=H \cap G_{w_{i}}=H \cap g_{i} A g_{i}^{-1}=1,
$$

while from the last two

$$
k_{2}^{-1} k_{1}=g_{i} g_{\mu(2)} a_{4} g_{s(2)}^{-1} g_{s(1)} a_{2}^{-1} g_{\mu(1)}^{-1} g_{i}^{-1} \in K \cap g_{i} G_{j} g_{i}^{-1}=K \cap G_{w_{i}}=K \cap g_{i} A g_{i}^{-1}=1 \text {. }
$$

The above relations imply that $g_{\lambda(2)} a_{3} g_{s(2)}^{-1} g_{s(1)} a_{1}^{-1} g_{\lambda(1)}^{-1}=1$ and $g_{\mu(2)} a_{4} g_{s(2)}^{-1} g_{s(1)} a_{2}^{-1} g_{\mu(1)}^{-1}=$ 1. Thus, $g_{\lambda(1)} g_{\mu(1)}^{-1}=g_{\lambda(2)} a_{3} g_{s(2)}^{-1} g_{s(1)} a_{1}^{-1} a_{2} g_{s(1)}^{-1} g_{s(2)} a_{4}^{-1} g_{\mu(2)}^{-1}$, from which it follows that $\phi\left(g_{\lambda(1)}\right) \cdot \phi\left(g_{\mu(1)}\right)^{-1}=\phi\left(g_{\lambda(2)}\right) \cdot \phi\left(g_{\mu(2)}\right)^{-1}$.

Our aim is to apply [6, Corollary 3.5], which requires pairwise-disjoint, single-quotient subsets. Note that if the intersection $C_{k} \cap C_{s}$ is nonempty, then there are edges $y_{1}$ and $y_{2}$ in $\operatorname{Star}_{X}\left(v_{k}\right)$ and $\operatorname{Star}_{X}\left(v_{s}\right)$, respectively, such that $\pi\left(y_{1}\right)=\pi\left(y_{2}\right)$. Thus, for each $k=1, \ldots, n$, we choose a subset $F_{k}$ of $\operatorname{Star}_{X}\left(v_{k}\right)$ with $\left|F_{1}\right|+\cdots+\left|F_{n}\right|$ maximum such that 
the restriction of $\pi$ on the union $\cup_{k=1}^{n} F_{k}$ is an injection. In particular, they are pairwisedisjoint. Since the inverse image of any edge of $Y \times Z$ under $\pi$ contains at most $N$ elements, we have $\left|\operatorname{Star}_{X}\left(v_{1}\right)\right|+\cdots+\left|\operatorname{Star}_{X}\left(v_{n}\right)\right| \leq N\left(\left|F_{1}\right|+\cdots+\left|F_{n}\right|\right)$. If $C_{F(k)}$ denotes the subset of $C_{k}$ corresponding to edges of $F_{k}$, then $C_{F(1)}, \ldots, C_{F(n)}$ are pairwise-disjoint. It follows that $\phi\left(C_{F(1)}\right), \ldots, \phi\left(C_{F(n)}\right)$ are pairwise-disjoint, single-quotient subsets of $\phi\left(\mathcal{R}_{H}\right) \times \phi\left(\mathcal{R}_{K}\right)$ and [6, Corollary 3.5] applies to show that

$$
\sum_{k=1}^{n}\left(\left|\phi\left(C_{F(k)}\right)\right|-2\right) \leq \theta\left(G_{j} / A\right) \cdot\left(\left|\phi\left(\mathcal{R}_{H}\right)\right|-2\right) \cdot\left(\left|\phi\left(\mathcal{R}_{K}\right)\right|-2\right) .
$$

Finally

$$
\begin{aligned}
\sum_{k=1}^{n}\left(\operatorname{deg}_{\widetilde{X}}\left(v_{k}\right)-2\right) & =\sum_{k=1}^{n}\left(\operatorname{deg}_{X}\left(v_{k}\right)-2\right)=\sum_{k=1}^{n}\left|\operatorname{Star}_{X}\left(v_{k}\right)\right|-2 n \leq N \cdot \sum_{k=1}^{n}\left|F_{k}\right|-2 N \\
& =N \cdot \sum_{k=1}^{n}\left(\left|C_{F(k)}\right|-2\right)=N \cdot \sum_{k=1}^{n}\left(\left|\phi\left(C_{F(k)}\right)\right|-2\right) \\
& \leq N \cdot \theta\left(G_{j} / A\right) \cdot\left(\left|\phi\left(\mathcal{R}_{H}\right)\right|-2\right) \cdot\left(\left|\phi\left(\mathcal{R}_{K}\right)\right|-2\right) \\
& =N \cdot \theta\left(G_{j} / A\right) \cdot\left(\left|\mathcal{R}_{H}\right|-2\right) \cdot\left(\left|\mathcal{R}_{K}\right|-2\right) \\
& \leq N \cdot \theta \cdot\left(\operatorname{deg}_{\widetilde{Y}}(a)-2\right) \cdot\left(\operatorname{deg}_{\widetilde{Z}}(b)-2\right) .
\end{aligned}
$$

This completes the proof.

Remark 3.7. The analogous theorem with the same proof is valid for fundamental groups of graphs of groups $(\mathcal{G}, \Psi)$ defined as follows. The subject graph $\Psi$ is the same as the one defined previously (prior to Theorem 3.6). To the terminal vertex $u_{i}$ of $e_{i}$ we associate the group $G_{i}$, to the common initial vertex of $e_{i}$ 's we associate the finite group $A$, and to each open edge $e_{i}$ we associate a subgroup $A_{i}$ of $A$ normally embedded in $G_{i}$ such that $A_{i_{0}}=A$ for some $i_{0}$ (this means that the "central" vertex is $G$-degenerate and thus $G_{w_{i}}=g A g^{-1}$ in Case (i) of the proof). To each loop we associate the trivial group. We need normality of $A_{i}$ in $G_{i}$ in order to make the natural map $G_{i} \rightarrow G_{i} / A_{i}$ a homomorphism (and thus the same arguments in Case (ii) work equally well to this more general setting).

As a corollary, we obtain the main result of Ivanov in [11] (in fact our proof can be slightly modified to generalize [6, Theorem 6.3] as well).

Corollary 3.8. Suppose that $H_{1}, H_{2}$ are subgroups of a free product $G=*_{a \in I} G_{a}$ and $H_{1}$, $\mathrm{H}_{2}$ have finite Kurosh rank $K\left(H_{1}\right), K\left(H_{2}\right)$. Then the intersection $H_{1} \cap H_{2}$ also has finite Kurosh rank and

$$
\bar{K} r\left(H_{1} \cap H_{2}\right) \leq 2 \cdot \theta(G) \cdot \bar{K} r\left(H_{1}\right) \cdot \bar{K} r\left(H_{2}\right) .
$$

In particular, if $G$ is torsion-free (or more generally, every finite subgroup of $G$ has order at most 2), then

$$
\bar{K} r\left(H_{1} \cap H_{2}\right) \leq 2 \cdot \bar{K} r\left(H_{1}\right) \cdot \bar{K} r\left(H_{2}\right) .
$$


Proof. By Lemma 2.3 and the comments preceding it, the subgroup Kurosh rank is equal to the Kurosh rank with respect to $T$ (i.e. $K r(\cdot)=K_{T}(\cdot)$, where $T$ is as above) and finite Kurosh rank implies tameness.

In the case of free products with a finite, normal subgroup amalgamated, we can use the same arguments to improve the bound for the complexity of the intersection of tame subgroups.

Let $G_{i}, i \in I$, be a family of groups together with a group $A$ and let $G=*_{A} G_{i}$ be the amalgamated free product of $G_{i}$ 's with amalgamated subgroup $A$ (with respect to a family of monomorphisms, regarded as inclusions). We construct a tree of groups $\left(\mathcal{G}, T_{0}\right)$ with fundamental group $G$ as usual. The tree $T_{0}$ consists of a wedge of open edges $e_{i}=\left[u_{0}, u_{i}\right], i \in I$ (one for each factor $G_{i}$ ) attached at a vertex $v_{0}$ (where $0 \notin I$ ) with vertex group $A$. To each edge we associate the group $A$ and to each vertex $v_{i}$ we associate the group $G_{i}$. We denote by $T$ the corresponding universal tree.

Theorem 3.9. Let $G=*_{A} G_{i}$ be the amalgamated free product of $G_{i}$ 's with a finite and normal amalgamated subgroup $A$. We consider the action of $G$ on $T$ defined above. If $H$ and $K$ are tame subgroups (with respect to $T$ ) of $G$, then $H \cap K$ is tame and

$$
\bar{C}_{T}(H \cap K) \leq 2 \cdot \theta \cdot|A \cap H K| \cdot \bar{C}_{T}(H) \cdot \bar{C}_{T}(K),
$$

where $\theta=\max \left\{\theta\left(G_{i} / A\right): i \in I\right\}$.

Proof. The proof is exactly the same as the proof of Theorem 3.6. There are two things to note:

(a) The normality of $A$ in $G$ and the fact that $v_{0}$ is a $G$-degenerate vertex imply that for each subgroup $B$ of $G$ the $B$-stabilizer of the star of any $B$-degenerate vertex $v$ of $T$ is equal to $B_{v}$ and therefore Lemma 3.2 (2) applies (i.e we can again use 1 instead of $M$ to obtain inequality 12).

(b) Using the notation of the proof of Theorem 3.6, the relations 13 and 14 now give $g_{\lambda(2)} a_{3} g_{s(2)}^{-1} g_{s(1)} a_{1}^{-1} g_{\lambda(1)}^{-1} \in A$ and $g_{\mu(2)} a_{4} g_{s(2)}^{-1} g_{s(1)} a_{2}^{-1} g_{\mu(1)}^{-1} \in A$. Since $A$ is the kernel of $\phi$, we again conclude that $\phi\left(g_{\lambda(1)}\right) \cdot \phi\left(g_{\mu(1)}\right)^{-1}=\phi\left(g_{\lambda(2)}\right) \cdot \phi\left(g_{\mu(2)}\right)^{-1}$.

Remark 3.10. In general, there are examples (see [11, 16]) showing that the bounds obtained in the previous two theorems are sharp. 


\section{References}

[1] Y. Antolín, A. Martino and I. Schwabrow, Kurosh rank of intersections of subgroups of free products of right-orderable groups, Math. Res. Lett. 21, No. 4 (2014), 649-661.

[2] V. Araújo, P. V. Silva and M. Sykiotis. Finiteness results for subgroups of finite extensions, J. Algebra 423 (2015), 592-614.

[3] H. Bass, Covering theory for graphs of groups, J. Pure Appl. Algebra 89 (1993), no. $1-2,3-47$.

[4] R. G. Burns, T. C. Chau and S.-M. Kam, On the rank of intersection of subgroups of a free product of groups, J. Pure App. Algebra 124 (1998), 31-45.

[5] W. Dicks and M.J. Dunwoody, "Groups acting on graphs", Cambridge University Press, 1989.

[6] W. Dicks and S. V. Ivanov, On the intersection of free subgroups in free products of groups, Math. Proc. Cambridge Phil. Soc. 144 (2008), 511-534.

[7] W. Dicks and S. V. Ivanov, On the intersection of free subgroups in free products of groups with no 2-torsion, Illinois J. Math. 54 (2010), 223-248.

[8] J. Friedman, Sheaves on graphs, their homological invar iants, and a proof of the Hanna Neumann conjecture: with an appendix by Warren Dicks, Memoirs Amer. Math. Soc. 233, no. 1100 (2014).

[9] A. G. Howson, On the intersection of finitely generated free groups, J. London Math. Soc. 29 (1954), 428-434.

[10] S. V. Ivanov, Intersecting free subgroups in free products of groups. Internat. J. Algebra Comput. 11 (2001), no. 3, 281-290.

[11] S. V. Ivanov, On the Kurosh rank of the intersection of subgroups in free products of groups, Adv. Math. 218 (2008), no. 2, 465-484.

[12] I. Mineyev, Submultiplicativity and the Hanna Neumann conjecture. Ann. of Math. (2) 175 (2012), 393-414.

[13] J.-P. Serre, "Trees", Springer-Verlag, Berlin-Heidelberg-New York, 1980.

[14] T. Soma, Intersection of finitely generated surface groups. J. Pure Appl. Algebra 66 (1990), no. 1-3, 81-95. 
[15] M. Sykiotis, On subgroups of finite complexity in groups acting on trees, J. Pure Appl. Algebra 200 (2005), 1-23.

[16] A. Zakharov, On the rank of the intersection of free subgroups in virtually free groups, J. Algebra 418 (2014), 29-43.

[17] A. Zakharov, Intersecting free subgroups in free amalgamated products of two groups with normal finite amalgamated subgroup, Mat. Sb. 204 (2) (2013), 223236.

Department of Mathematics

National and Kapodistrian University of Athens

Panepistimioupolis, GR-157 84, Athens, Greece

e-mail: klentzos@math.uoa.gr

e-mail: msykiot@math.uoa.gr 